\title{
Formation and Structure of Transport Barriers During Confinement Transitions in Toroidal Plasma
}

\author{
H. Punzmann* and M. G. Shats ${ }^{\dagger}$ \\ Plasma Research Laboratory, Research School of Physical Sciences and Engineering, Australian National University, \\ Canberra ACT 0200, Australia
}

(Received 3 August 2003; published 15 September 2004)

\begin{abstract}
Density pedestal formation is studied experimentally during spontaneous low-to-high confinement transitions in the H-1 heliac. Poloidally extended potential structures, or zonal flows, seem to play the major role both in the spatial structure and in the temporal evolution of the pedestal formation. Zonal flows transiently generate radially localized maxima in the radial electric-field shear in $L$ mode which coincides with the radial location of the pedestal in $H$ mode.
\end{abstract}

DOI: 10.1103/PhysRevLett.93.125003

Fast, sudden changes in plasma confinement have been studied for more than two decades, since the discovery of the low-to-high ( $L$ to $H$ ) mode transitions in the ASDEX tokamak [1]. Such transitions, which were later observed in a variety of experiments, are characterized by the formation of transport barriers and radial plasma regions where transport properties of plasma are significantly improved (for review see [2,3]). In tokamaks, these are referred to as edge pedestals. Understanding and predicting the pedestal characteristics has become a focus of the international fusion community in view of its importance for the performance of future reactor-relevant magnetic fusion devices (see, e.g., [4,5]). However the physics of the transport barrier formation remains far from being fully understood.

In this Letter we present the first experimental results on the spatiotemporal structure of the transport barrier across $L-H$ bifurcations in the $\mathrm{H}-1$ heliac. It is shown that a density pedestal similar to those observed in tokamaks develops during the $L-H$ transitions in the $\mathrm{H}-1$ heliac. Detailed measurements of the temporal evolution and the spatial structure of spontaneous transitions from $L$ to $H$ mode reveal that the top of the pedestal, which develops in $H$ mode, coincides with the radial region where the largest time-varying $E_{r}$ shear developed prior to the transition, in $L$ mode. These oscillations in the $E_{r}$ shear are found to be due to strong zonal flows which are observed in discharges with spontaneous $L-H$ transitions. These findings point to the idea of the zonal flow acting as a seed, or a nucleus, from which $H$ mode develops across the transition.

We report experiments performed in the $\mathrm{H}-1$ toroidal heliac [6], a 3-field-period helical axis stellarator, which has a major radius of $R=1 \mathrm{~m}$ and a mean minor radius of $\bar{a} \approx 0.2 \mathrm{~m}$. Plasma is produced by $\sim 60 \mathrm{~kW}$ of radiofrequency waves at $7 \mathrm{MHz}$ at low magnetic field in the range of $B=(0.05-0.15)$ T. Plasma parameters are as follows: $n_{e} \approx 1 \times 10^{18} \mathrm{~m}^{-3}, T_{e} \approx 10 \mathrm{eV}, T_{i} \approx 40 \mathrm{eV}$ in argon at filling pressure of $\sim 3 \times 10^{-5}$ Torr.

Sudden changes in the particle confinement observed in such H-1 conditions [7-9] show many features similar to
PACS numbers: 52.25.Fi, 52.25.Gj, 52.55.-s

the $L-H$ transitions in tokamaks. The list of known similarities includes a large improvement in the energy or particle confinement, steeper plasma pressure profiles in $H$ mode, an increase in the radial electric-field shear across the transition, and suppression of fluctuations and of the associated anomalous transport. In this paper we add one more characteristic, namely, the development of the density pedestal in $H$ mode. The transition dynamics of spontaneous $L-H$ transitions in the $\mathrm{H}-1$ heliac [10] appear to be similar to that of slow $L-H$ transitions in the DIII-D tokamak $[11,12]$. In both cases plasma spontaneously bifurcates from a turbulence-dominated $L$ mode state into a quiescent $H$ mode. Despite large differences between plasma parameters in $\mathrm{H}-1$ at low magnetic fields and the edge plasma in large tokamaks, these experiments appear to be dimensionally similar, as will be discussed later.

Diagnostics used in the reported experiments include a multichannel spectroscopy setup [13] to study the spatial structure of the particle transport, and Langmuir probe arrays, capable of measuring local electron density, electron temperature, radial electric fields and their fluctuations, including poloidal and toroidal wave numbers. In the spectroscopy diagnostic, visible light is collected from ten radial chords at two wavelengths: $\lambda=763.5 \mathrm{~nm}$ (ArI excited neutral line) and $\lambda=488.0 \mathrm{~nm}$ (ArII excited ion line). Simultaneous measurements of neutral and ion emissivities combined with $T_{e}$ measurements using triple probes [14] allow the electron density $n_{e}$, the neutral density $n_{0}$, and the particle source due to the electron impact ionization $S=n_{e} n_{o}\left\langle\sigma v_{e}\right\rangle_{i}$ to be recovered. The net particle flux $\Gamma$ is determined using the continuity equation $\partial n / \partial t=$ $-\nabla \cdot \Gamma+S$ by analyzing steady state discharges in which $\partial n / \partial t \approx 0$. For slow processes, when $S \gg \partial n / \partial t$, the particle flux is estimated as

$$
\Gamma(r)=\frac{1}{r} \int_{0}^{r} r S d r=\frac{1}{r} \int_{0}^{r} r n_{e}(r) n_{0}(r)\left\langle\sigma v_{e}\right\rangle_{i} d r .
$$

Figure 1(a) shows the time evolution of the mean density 


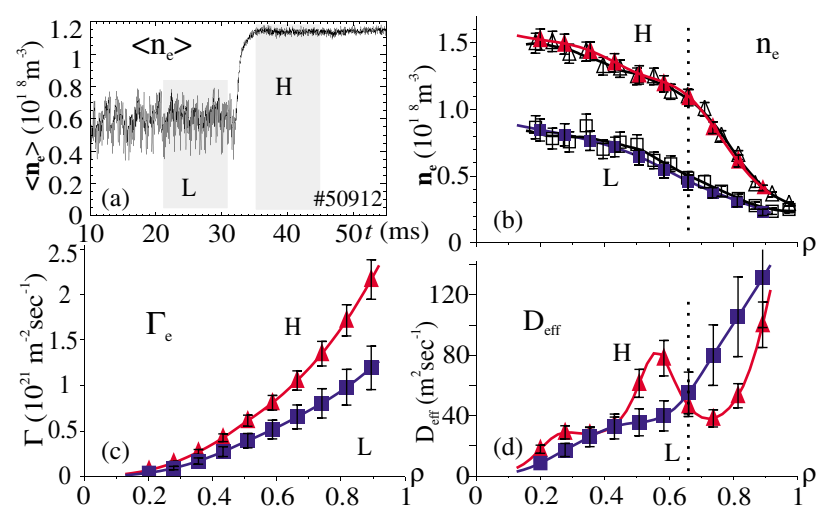

FIG. 1 (color online). (a) Temporal evolution of mean electron density and radial profiles of (b) electron density derived from spectroscopy (solid symbols) and probes (open symbols), (c) electron particle flux, and (d) effective diffusion coefficient during spontaneous transition from $L$ mode (squares) to $H$ mode (triangles).

in a discharge which exhibits a spontaneous confinement transition from $L$ to $H$ mode. The electron density increases by a factor of 2 in about $1 \mathrm{~ms}$. Such spontaneous transitions are remarkably reproducible in $\mathrm{H}-1$. The timing of the transition during the discharge can be controlled (to within $\pm 5 \mathrm{~ms}$ ) by tuning operational parameters (e.g., magnetic field, heating power, and neutral gas fill pressure). Equilibrium profiles for $L$ and $H$ modes in Fig. 1(b)-1(d) have been averaged over the $10 \mathrm{~ms}$ time intervals, highlighted in gray in Fig. 1(a). Figure 1(b) shows the reconstructed electron density profiles using the spectroscopy (solid symbols). Superimposed are the electron density profiles determined from a radial scan using triple probes (open symbols). The spectroscopy and probe profiles are in good agreement and show a noticeable change of the $H$ mode density gradient at around $\rho_{\text {ped }}=r / a \approx 0.67$. Because of the distinct change in density gradient this point is identified as the top of the $H$ mode pedestal.

Figures 1(c) and 1(d) show time-averaged radial profiles of the net electron particle flux and the effective diffusion coefficient, $D_{e f f}=\Gamma / \nabla n$ in both $L$ and $H$ confinement modes. Across the bifurcation the net particle flux increases in $H$ mode and the density profiles steepen within the pedestal region of $0.67<\rho<1.0$ as seen in Fig. 1(b). As a result, the structure of the particle transport around $\rho_{\text {ped }}$ shows a substantial decrease in $D_{\text {eff }}$ in the pedestal region owing to the improvement in particle confinement. Inside $\rho<\rho_{\text {ped }}$ however, confinement deteriorates because net fluxes are usually higher in $H$ mode while the gradients are comparable to those in $L$ mode. In a narrow radial region around the top of the pedestal $D_{\text {eff }}$ remains constant across the bifurcation as seen in Fig. 1(d).

Experimental errors in the estimation of the source term as well as in the determination of $D_{\text {eff }}$ can be separated into systematic and statistical errors, similar to those discussed in [15]. Statistical errors are estimated as standard deviations of measured and derived plasma parameters averaged over 12 discharges. In the case of the electron density [Fig. 1(b)] this error is below $10 \%$ for $L$ mode and $5 \%$ for $H$ mode. Statistical error bars in Fig. 1(c) and 1(d) are $20 \%$ for the fluxes and $25 \%$ for the diffusion coefficient in $L$ mode and $10 \%$ and $15 \%$, respectively, for $H$ mode. Systematic errors originate from the relative spatial channel calibration of the spectroscopy diagnostic, inversion of the chord averaged emissivities, and the probe measurements of the electron temperature. These systematic errors are estimated as follows: $15 \%$ error in the electron density, $50 \%$ error in the flux, and up to $80 \%$ error in the diffusion coefficient. In Fig. 1(b)-1(d) we compare profiles of plasma parameters obtained within the same discharge in $L$ and $H$ mode. The error bars shown in Fig. 1 therefore only refer to statistical errors in the measurements.

Plasma potentials and the electron density show strong oscillations prior to the $L-H$ transition. In the discharges with spontaneous transitions these oscillations have been identified as zonal flows [16]. Typically, their frequency is in the range of $4-8 \mathrm{kHz}$, though lower-frequency zonal flows ( $\approx 1 \mathrm{kHz}$ ) have also been observed. $\tilde{E}_{r}$ fluctuations measured using two radially separated probes are affected by the potential fluctuations having finite $k_{r}$. However only poloidally extended zonal flows which have $k_{r} \gg k_{\theta} \simeq 0$ generate nonzero flux-surface-averaged fluctuations in the radial electric field $\left\langle\tilde{E}_{r}\right\rangle_{\theta}$. A zonal-flow identification requires simultaneous measurements of the wave numbers $k_{\theta}$ and $k_{r}$ in the plasma potential fluctuations, as described in detail in [16]. Such measurements were performed in $\mathrm{H}-1$ at the plasma midplane and have also been verified at $\pm 15 \mathrm{~cm}$ from the midplane. The analysis shows that only fluctuations at the frequency of $5.6 \mathrm{kHz}$ are poloidally extended structures with $k_{\theta} \simeq$ $2 \mathrm{~m}^{-1}$, as is seen in the floating potential spectrum of Fig. 2(b). For all other spectral features $k_{\theta}$ is well above $10 \mathrm{~m}^{-1}$. Therefore only the potential structure at $5.6 \mathrm{kHz}$ does not average on the flux surface and produces fluctuations in the radial electric field $\tilde{E}_{r}$. The radial profile of $\tilde{E}_{r}$ at $f_{Z F}=5.6 \mathrm{kHz}$ along with its phase are shown in Fig. 2(c). Oscillations at the zonal-flow frequency also dominate the density fluctuation spectrum as seen in Fig. 2(a). The ion saturation current $\left(I_{s} \sim n_{e}\right)$ used here is measured at $\rho \approx 0.6$.

A time trace of $\tilde{E}_{r}$ at the zonal-flow frequency in $L$ mode prior to the transition is shown in Fig. 3(a). The relative peak-to-peak fluctuation level of $\tilde{E}_{r^{p-p}} / \bar{E}_{r} \approx 1.7$ at $\rho \approx 0.6$. The amplitude and phase profiles of $\tilde{E}_{r}$ in Fig. 2(c) are used to reconstruct time-resolved radial profiles of the radial electric field in $L$ mode by superimposing $\tilde{E}_{r}$ on the time-averaged $\bar{E}_{r}$ profiles. These mean $\bar{E}_{r}$ profiles are shown in Fig. 3(b) (solid lines) together 


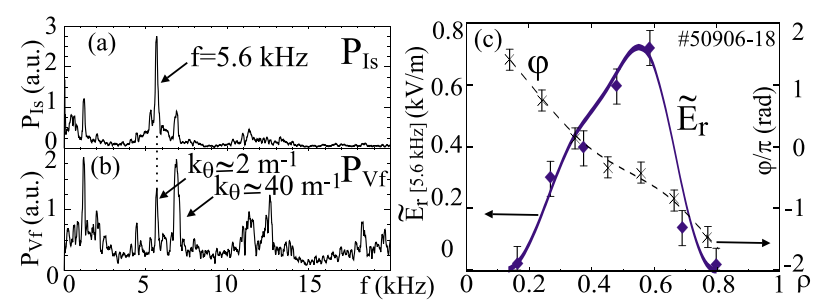

FIG. 2 (color online). Power spectrum of (a) ion saturation current $\left(\propto n_{e}\right)$ and (b) plasma floating potential. The $f_{Z F}=$ $5.6 \mathrm{kHz}$ feature is a zonal flow with $k_{\theta} \simeq 2 \mathrm{~m}^{-1}$. (c) The profile of the $\tilde{E}_{r}$ fluctuation amplitude and phase of the zonal flow, measured during a radial probe scan.

with the envelope of the zonal-flow-produced $E_{r}$ fluctuations, shown within the gray shaded area. Dotted lines represent the fluctuation envelope. Shown in Fig. 3(c) are the profiles of $\bar{E}_{r}{ }^{\prime}$ along with the fluctuation envelope in $L$ mode, as well as $\bar{E}_{r}{ }^{\prime}$ in $H$ mode, derived from the profiles of Fig. 3(b). The maximum of the $\tilde{E}_{r}{ }^{\prime}$ envelope [dotted lines in Fig. 3(c)] is located at $\rho \approx 0.67$. At this location the amplitude of $\tilde{E}_{r}{ }^{\prime}$ in $L$ mode prior to the bifurcation is comparable to the mean $\bar{E}_{r}$ shear in $H$ mode. It is about this radial position where the $H$ mode pedestal at $\rho_{\text {ped }} \approx$ 0.67 develops after the confinement transition.

The top of the pedestal in $H$ mode seen in Fig. 1(b) is characterized by a radially localized maximum in the second derivative of the electron density, seen as a kink in the $\mathrm{n}_{e}$ profile at $\rho_{\text {ped }} \approx 0.67$. To understand why the

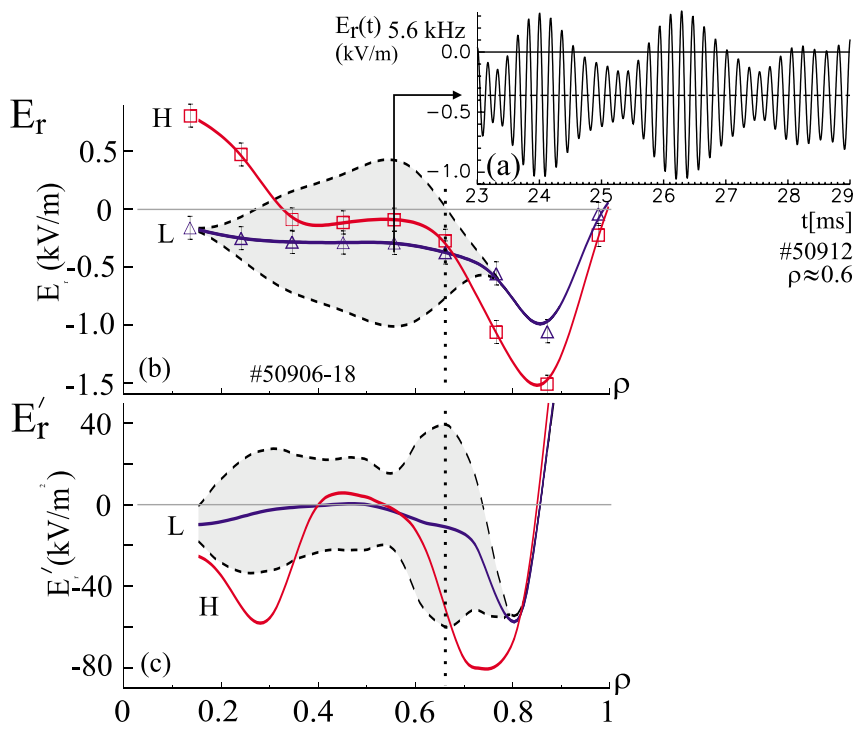

FIG. 3 (color online). (a) Time-varying radial electric-field fluctuations at the zonal-flow frequency $f_{Z F}=5.6 \mathrm{kHz}$. (b) Radial profiles of the mean radial electric field $\bar{E}_{r}$ in $L$ mode (triangles), and $H$ mode (squares), and $E_{r}$ fluctuations at $f_{Z F}=5.6 \mathrm{kHz}$ (gray shaded area within dashed lines). (c) Derived radial electric-field shear for both mean and time-varying $E_{r}{ }^{\prime}$ in $L$ mode and mean $E_{r}{ }^{\prime}$ in $H$ mode. maximum of $E_{r}$ shear in $L$ mode coincides with the position of the top of the pedestal which develops during the transition to $H$ mode, we use the radial force balance arguments. It has been shown in [8] that in $\mathrm{H}-1$, poloidal and toroidal ion flow velocities are negligibly small compared with the $E \times B$ drift velocity, so that the radial force balance equation is reduced to the balance between the radial electric field and the ion pressure gradient term $E_{r} \approx \nabla\left(n T_{i}\right) /(e n)$. It has also been shown in [8] that the ion temperature profiles are relatively flat in both $L$ and $H$ modes. Assuming $\partial T_{i} / \partial r \approx 0$, and by radially differentiating the above equation we obtain for the $E_{r}$ shear:

$$
E_{r}^{\prime}=\left(T_{i} / e n\right)\left[\partial^{2} n / \partial r^{2}-(\partial n / \partial r)^{2} / n\right] .
$$

In the following interpretation we neglect $T_{i}$ fluctuations. The second term in the square brackets on the right-hand side is always negative, so that the time variation in $E_{r}^{\prime}$ in the range between -60 to $+40 \mathrm{kV} / \mathrm{m}^{2}$ (seen in Fig. 3(c) at $\rho \approx 0.67$ ) can only occur if the second derivative $\partial^{2} n / \partial r^{2}$ at least transiently dominates over the $(\partial n / \partial r)^{2} / n$ term. Thus the time evolution of the $E_{r}$ shear is suggestive of the transient development of a strong second derivative in the electron density at $\rho_{\text {ped }} \approx 0.67$ (the kink in the density profile) even in $L$ mode, before the $L-H$ transition.

It is possible that this transient kink at $\rho_{\text {ped }}$ is then secured during the $L-H$ transition when the fluctuations are suppressed. Thus one might speculate that the radial localization of zonal flows in $L$ mode, before the transition predetermines the location of the top of the pedestal in $H$ mode.

In addition, a zonal flow precedes the $L-H$ transition in the time domain [see Fig. 3(a)]. In similar plasma conditions in $\mathrm{H}-1$ [10] it was reported that strong, low frequency oscillations in the electron density precede the confinement bifurcation. It has also been shown in [10] that these oscillations have $m=n=0$ poloidal and toroidal mode numbers, as one would expect for the zonalflow-produced density fluctuations.

This observation is not unique to H-1. Colchin et al. $[11,12]$ refer to similar oscillations observed in the DIII$\mathrm{D}$ experiment as an intermediate confinement regime ( $I$ mode) between $L$ and $H$ modes and associate them with the self-generated shear flows, though no experimental proof of the zonal-flow presence in $I$ mode was given. Similar dynamics has been discovered in a theoretical model [17] convincingly illustrating the role of the following main ingredients in the transition: zonal flows, mean $E \times B$ flows, and the ion pressure gradient. Similar to our observations it has been demonstrated in [17] that plasma parameters oscillate at the zonal-flow frequency before the $L-H$ transition. The role of zonal flows in the temporal dynamics of the $L-H$ transitions has also been confirmed indirectly in $[18,19]$. The nonlinear coupling between small scale and larger scale lower-frequency 
fluctuations was found to increase transiently before and during the spontaneous $L-H$ transitions in the DIII-D tokamak [19]. It was argued that such an increase was consistent with expectations for a transient Reynolds stress-driven zonal flow triggering the $L-H$ transition. All these observations point to the possibility that the turbulence-driven sheared flow acts as a $L-H$ transition trigger.

It should be noted that the role of zonal flows as an important factor in the $L-H$ transition dynamics has only been confirmed during spontaneous $L-H$ transitions in $\mathrm{H}-$ 1 as well as in DIII-D. When plasma is sufficiently close to the bifurcation point, zonal flows may act as a transition trigger by increasing the sheared radial electric field above the threshold necessary for the shear decorrelation of turbulent eddies. If transitions are induced, for example, by an external electrode biasing, or by a sudden increase in the heating power, the plasma may skip the zonal-flow development stage (or $I$ mode, according to [11]) during its transition to $H$ mode.

Despite large differences in plasma parameters (the electron temperature, density, and magnetic fields), plasmas in $\mathrm{H}-1$ and at the pedestal regions in large tokamaks, are dimensionally similar. Plasmas are referred to as dimensionally similar if several essential nondimensional parameters are the same $[20,21]$. Thus, a sizeable radial region of the low temperature plasma in $\mathrm{H}-1$ is dimensionally similar to a narrow region in the tokamak edge where transport barriers form during the $L-H$ transition. The pedestal width in $\mathrm{H}-1$ is about $\Delta r=0.06 \mathrm{~m}$ or $\Delta \rho=0.3$, while, for example, in the DIII-D tokamak [22] this corresponds to $\Delta r \leq 0.015 \mathrm{~m}$ and $\Delta \rho \leq 0.05$. Several nondimensional parameters are similar in the pedestal regions of DIII-D and H-1: the ion gyroradius normalized by the density scale length $\left(L_{n}=n_{e} / \nabla n_{e}\right)$ is $\rho *=\rho_{i} / L_{n}=(0.4-1)$ in both machines; the ratio of the plasma pressure to the magnetic pressure is $\beta \approx 0.01$; the relative electron collisionality $\nu_{e}^{*}$, defined as the ratio of the effective collision rate $\nu_{e i}^{e f f}$ to the bounce frequency $\omega_{b e}$ of trapped particles, is about unity $\nu_{e}^{*} \approx 1$ in both experiments in the vicinity of the pedestal. The ranges for these three nondimensional parameters are comparable.

Summarizing, we report the first observation of the density pedestal in $H$ mode in the $\mathrm{H}-1$ heliac and show detailed measurements of the $L-H$ transition dynamics leading to the transport barrier generation. Presented results emphasize the role played by zonal flows in the physics of the pedestal formation during spontaneous $L-H$ transitions. In particular, the radial region of the maximum in the $E_{r}$ shear, which transiently develops in the presence of zonal flows in $L$ mode, coincides with the position of the top of the density pedestal in $H$ mode. Zonal flows also dominate the temporal evolution of the plasma parameters before the bifurcation and possibly act as a trigger for the $L-H$ transition.

The role played by zonal flows in the confinement transitions suggests the analogy with the physics of phase transitions [23]. Spontaneous $L-H$ transitions can be likened to transitions from the metastable state (e.g., supersaturated gas) to a new stable state (liquid). In these transitions the nucleation process can play an important role, e.g., a growth of the supercritical nucleus, such as a liquid droplet in a gas. In plasma, a zonal flow, which is a turbulence-generated time-varying sheared flow, is, by its nature, a nucleus of the new stable state (sheared-radialelectric-field-dominated $H$ mode) in the turbulencedominated $L$ mode, or, more precisely, in the metastable I mode. More studies are necessary to further develop this analogy.

*Electronic address: Horst.Punzmann@anu.edu.au †Electronic address: Michael.Shats@anu.edu.au

[1] F. Wagner et al., Phys. Rev. Lett. 49, 1408 (1982).

[2] J.W. Connor and H. R. Wilson, Plasma Phys. Controlled Fusion 42, R1 (2000).

[3] A. Fujisawa, Plasma Phys. Controlled Fusion 45, R1 (2003).

[4] T. Hatae et al., Nucl. Fusion 41, 285 (2001).

[5] T. Fujita, Plasma Phys. Controlled Fusion 44, A19 (2002).

[6] S. M. Hamberger et al., Fusion Technol. 17, 123 (1990).

[7] M. G. Shats et al., Phys. Rev. Lett. 77, 4190 (1996).

[8] M. G. Shats et al., Phys. Plasmas 4, 3629 (1997).

[9] M. G. Shats et al., Phys. Plasmas 5, 2390 (1998).

[10] D. L. Rudakov et al., Plasma Phys. Controlled Fusion 43, 559 (2001).

[11] R. J. Colchin et al., Phys. Rev. Lett. 88, 255002 (2002).

[12] R. J. Colchin et al., Nucl. Fusion 42, 1134 (2002).

[13] H. Punzmann et al., Rev. Sci. Instrum. 74, 2048 (2003).

[14] M. G. Shats, Plasma Phys. Controlled Fusion 41, 1357 (1999).

[15] J. O'Rourke et al., Plasma Phys. Controlled Fusion 35, 585 (1993).

[16] M. G. Shats and W. M. Solomon, Phys. Rev. Lett. 88, 045001 (2002).

[17] E. J. Kim and P. H. Diamond, Phys. Rev. Lett. 90, 185006 (2003).

[18] G. R. Tynan et al., Phys. Plasmas 8, 2691 (2001).

[19] R. A. Moyer et al., Phys. Rev. Lett. 87, 135001 (2001).

[20] B. B. Kadomtsev, Fiz. Plazmy 1, 531 (1975).

[21] J.W. Connor and J. B. Taylor, Nucl. Fusion 17, 1047 (1977).

[22] T. N. Carlstrom and R. J. Groebner, Phys. Plasmas 10, 1867 (1996).

[23] L. D. Landau and E. M. Lifschitz, Statistical Physics, Part 1 (Pergamon Press, New York, 1968), p. 471. 